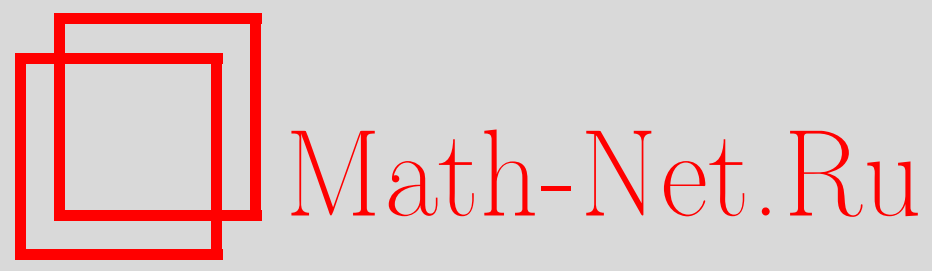

А. В. Лапшин, Распределение вероятностей сумм элементов на непересекающихся частях случайной перестановки, Дискрет. матем., 2005, том 17, выпуск 3, 5-11

DOI: https://doi.org/10.4213/dm111

Использование Общероссийского математического портала Math-Net.Ru подразумевает, что вы прочитали и согласны с пользовательским соглашением http://www.mathnet.ru/rus/agreement

Параметры загрузки:

IP: 54.197 .130 .99

26 апреля 2023 г., 12:24:11 


\title{
Распределение вероятностей сумм элементов на непересекающихся частях случайной перестановки
}

\author{
() 2005 г. А. В. Лапшин
}

\begin{abstract}
В работе исследуется совместное распределение вероятностей сумм элементов на непересекающихся множествах мест случайной перестановки из одного класса перестановок. Вычислена производящая функция распределения вероятностей таких сумм, найдено предельное распределение соответствующих нормированных случайных величин при неограниченном росте числа элементов перестановки.
\end{abstract}

\section{1. Постановка задачи и основные результаты}

Рассмотрим класс $n$-перестановок соответствующей конфигурации, в которой среди $n$ элементов содержится $n_{k}$ элементов $k$-го типа, $k=1, \ldots, d, n_{1}+\ldots+n_{d}=n$ (определения см. в [1]). Полагаем, что элементами $k$-го типа являются действительные числа $w_{k}$, $k=1, \ldots, d$. Обозначим $S$ множество перестановок рассматриваемого класса, мощность множества $S$ равна

$$
\frac{n !}{n_{1} ! \ldots n_{d} !}
$$

Пусть

$$
\left\{s\left(i_{1}^{(1)}\right), \ldots, s\left(i_{l_{1}}^{(1)}\right)\right\}, \quad\left\{s\left(i_{1}^{(2)}, \ldots, s\left(i_{l_{2}}^{(2)}\right)\right\}, \quad \ldots, \quad\left\{s\left(i_{1}^{(p)}, \ldots, s\left(i_{l_{p}}^{(p)}\right)\right\}\right.\right.
$$

$-p$ наборов элементов случайной перестановки $s \in S$, соответственно, на местах

$$
\left\{i_{1}^{(1)}, \ldots, i_{l_{1}}^{(1)}\right\}, \quad\left\{i_{1}^{(2)}, \ldots, i_{l_{2}}^{(2)}\right\}, \quad \ldots, \quad\left\{i_{1}^{(p)}, \ldots, i_{l_{p}}^{(p)}\right\} .
$$

Далее будем требовать выполнения условия

$$
\left\{i_{1}^{(v)}, \ldots, i_{l_{1}}^{(\nu)}\right\} \cap\left\{i_{1}^{(\mu)}, \ldots, i_{l_{2}}^{(\mu)}\right\}=\varnothing, \quad \nu, \mu=1, \ldots, p, \quad v \neq \mu .
$$

Полагаем, что перестановка $s$ выбирается из множества $S$ случайно равновероятно. Рассмотрим случайные величины

$$
\xi_{t}=s\left(i_{1}^{(t)}\right)+\ldots+s\left(i_{l_{t}}^{(t)}\right), \quad t=1, \ldots, p
$$


В работе изучается распределение вероятностей случайного вектора $\left(\xi_{1}, \ldots, \xi_{p}\right)$ и находится его предельное распределение при $n \rightarrow \infty$, эти результаты были анонсированы в [3].

Для исследования числовых характеристик распределения случайного вектора $\left(\xi_{1}, \ldots, \xi_{p}\right)$ найдем выражение для производящей функщия $f\left(x_{1}, \ldots, x_{p}, y_{1}, \ldots, y_{p}\right)$, определенной равенством

$$
\begin{aligned}
f\left(x_{1}, \ldots, x_{p}, y_{1}, \ldots, y_{p}\right)= & \sum_{l_{1}+\ldots+l_{p} \leqslant n} \sum_{\sigma_{1}, \ldots, \sigma_{p} \geqslant 0} \frac{n !}{\left(n-l_{1}-\ldots-l_{p}\right) ! l_{1} ! \ldots l_{p} !} \\
& \times \mathbf{P}_{l_{1}, \ldots, l_{p}}\left(\xi_{1}=\sigma_{1}, \ldots, \xi_{p}=\sigma_{p}\right) x_{1}^{\sigma_{1}} \ldots x_{p}^{\sigma_{p}} y_{1}^{l_{1}} \ldots y_{p}^{l_{p}},
\end{aligned}
$$

где $\mathbf{P}_{l_{1}, \ldots, l_{p}}\left(\xi_{1}=\sigma_{1}, \ldots, \xi_{p}=\sigma_{p}\right)$ - вероятность того, что случайные величины $\xi_{1}, \ldots, \xi_{p}$ принимают, соответственно, значения $\sigma_{1}, \ldots, \sigma_{p}$ при значениях $l_{1}, \ldots, l_{p}$ частей случайной подстановки $S$, равновероятно выбранной из множества $S$.

Теорема 1. Справедливо равенство

$$
f\left(x_{1}, \ldots, x_{p}, y_{1}, \ldots, y_{p}\right)=\prod_{k=1}^{d}\left(1+x_{1}^{w_{k}} y_{1}+\ldots+x_{p}^{w_{k}} y_{p}\right)^{n_{k}} .
$$

Следствие 1. Справедливы равенства

$$
\mathbf{E} \xi_{t}=\frac{1}{n} l_{t} \sum_{k=1}^{d} w_{k} n_{k}, \quad \mathbf{D} \xi_{t}=\frac{1}{n-1} l_{t}\left(1-l_{t}\right) B_{n}, \quad t=1, \ldots, p
$$

где

$$
B_{n}=\frac{1}{n} \sum_{k=1}^{d} w_{k}^{2} n_{k}-\frac{1}{n^{2}}\left(\sum_{k=1}^{d} w_{k} n_{k}\right)^{2}
$$

Элементы соответствующей корреляционной матрицы $R_{n}=\left(\rho_{i j}^{(n)}\right), i, j=1, \ldots, p$, имеют вид

$$
\rho_{i j}^{(n)}= \begin{cases}1 & \text { при } i=j \\ -\sqrt{l_{i} l_{j} /\left(\left(n-l_{i}\right)\left(n-l_{j}\right)\right)} & \text { при } i \neq j\end{cases}
$$

Далее рассмотрим предельные при $n \rightarrow \infty$ характеристики случайного вектора $\left(\xi_{1}, \ldots, \xi_{p}\right)$. Введем обозначение

$$
\bar{\xi}=\left(\frac{\xi_{1}-\mathbf{E} \xi_{1}}{\sqrt{\mathbf{D} \xi_{1}}}, \ldots, \frac{\xi_{p}-\mathbf{E} \xi_{p}}{\sqrt{\mathbf{D} \xi_{p}}}\right)
$$

Теорема 2. Пусть $n \rightarrow \infty, l_{t} / n \rightarrow \alpha_{t}, \alpha_{t}>0, t=1, \ldots, p$,

$$
\lim _{n \rightarrow \infty} \frac{1}{n} \sum_{k=1}^{d} w_{k} n_{k}=A, \quad \lim _{n \rightarrow \infty}\left(\frac{1}{n} \sum_{k=1}^{d} w_{k}^{2} w_{k}-\frac{1}{n^{2}}\left(\sum_{k=1}^{d} w_{k} n_{k}\right)^{2}\right)=B, \quad B>0 .
$$


Тогда случайный вектор $\bar{\xi}$ имеет в пределе р-мерное нормальное распределение с нулевым вектором средних и матрицей коррелячий $R_{n}=\left(\rho_{i j}\right), i, j=1, \ldots, p$, в которой

$$
\rho_{i j}= \begin{cases}1 & \text { npu } i=j, \\ -\sqrt{\alpha_{i} \alpha_{j} /\left(\left(1-\alpha_{i}\right)\left(1-\alpha_{j}\right)\right)} & \text { npu } i \neq j\end{cases}
$$

\section{2. Доказательства}

Доказательство теоремы 1. Пусть среди элементов $s\left(i_{1}^{(t)}\right), \ldots, s\left(i_{l}^{(t)}\right)$ случайно выбранной перестановки $s$ из $S$ число $w_{k}$ содержится $v_{k}^{(t)}$ раз, $k=1, \ldots, d, t=1, \ldots, p$, тогда

$$
\xi_{t}=\sum_{k=1}^{d} w_{k} v_{k}^{(t)}, \quad \sum_{k=1}^{d} v_{k}^{(t)}=l_{t}, \quad t=1, \ldots, p, \quad \sum_{t=1}^{p} v_{k}^{(t)} \leqslant n_{k}, \quad k=1, \ldots, d,
$$

тогда

$$
\xi_{t}=\sum_{k=1}^{d} w_{k} v_{k}^{(t)}, \quad \sum_{k=1}^{d} v_{k}^{(t)}=l_{t}, \quad t=1, \ldots, p, \quad \sum_{t=1}^{p} v_{k}^{(t)} \leqslant n_{k}, \quad k=1, \ldots, d,
$$

таким образом, распределение вектора $\left(\xi_{1}, \ldots, \xi_{p}\right)$ полностью определяется распределением $d p$-мерного вектора

$$
\bar{v}=\left(v_{1}^{(1)}, \ldots, v_{d}^{(1)}, \ldots, v_{1}^{(p)}, \ldots, v_{d}^{(p)}\right) .
$$

Для упрощения записи введем обозначения

$$
\begin{aligned}
\bar{v}^{(t)} & =\left(v_{1}^{(t)}, \ldots, v_{d}^{(t)}\right), & & t=1, \ldots, p, \\
\bar{n}^{(t)} & =\left(n_{1}^{(t)}, \ldots, n_{d}^{(t)}\right), & & t=1, \ldots, p,
\end{aligned}
$$

где целые неотрицательные числа $n_{k}^{(t)}, k=1, \ldots, d, t=1, \ldots, p$, удовлетворяют соотношениям

$$
\sum_{k=1}^{d} n_{k}^{(t)}=l_{t}, \quad t=1, \ldots, p, \quad \sum_{t=1}^{p} n_{k}^{(t)} \leqslant n_{k}, \quad k=1, \ldots, d
$$

и пусть $\bar{n}=\left(\bar{n}^{(1)}, \ldots, \bar{n}^{(p)}\right)$.

Тогда распределение вектора $\bar{v}$ задается вероятностями

$$
\begin{aligned}
\mathbf{P}(\bar{v}=\bar{n}) & =\frac{\prod_{t=1}^{p} l_{t} !\left(n-\sum_{t=1}^{p} l_{t}\right) !}{\prod_{t=1}^{p} \prod_{k=1}^{d} n_{k}^{(t)} ! \prod_{k=1}^{d}\left(n_{k}-\sum_{t=1}^{p} n_{k}^{(t)}\right) !} / \frac{n !}{\prod_{k=1}^{d} n_{k} !} \\
& =\frac{1}{n !}\left(n-\sum_{t=1}^{p} l_{t}\right) ! \prod_{t=1}^{p} l_{t} ! \prod_{k=1}^{d} \frac{n_{k} !}{\left(n_{k}-\sum_{t=1}^{p} n_{k}^{(t)}\right) ! \prod_{t=1}^{p} n_{k}^{(t)} !}
\end{aligned}
$$


В этой формуле в числителе стоит число $n$-перестановок с данным составом элементов, а в знаменателе число всех $n$-перестановок.

Пусть

$$
\bar{x}_{t}=\left(x_{t 1}^{n_{1}^{(t)}}, \ldots, x_{t d}^{n_{d}^{(t)}}\right), \quad t=1, \ldots, p .
$$

Рассмотрим производящую функцию случайного вектора $\bar{v}$

$$
f_{l_{1}, \ldots, l_{p}}\left(\bar{x}_{1}, \ldots, \bar{x}_{p}\right)=\sum_{\bar{n}} \mathbf{P}(\bar{v}=\bar{n}) x_{11}^{n_{1}^{(1)}} \ldots x_{1 d}^{n_{d}^{(1)}} \ldots x_{p 1}^{n_{1}^{(p)}} \ldots x_{p d}^{n_{d}^{(p)}} .
$$

Введем функцию

$$
\begin{aligned}
f\left(\bar{x}_{1}, \ldots, \bar{x}_{p}, y_{1}, \ldots, y_{p}\right) & \\
& =\sum_{l_{1}+\ldots+l_{p} \leqslant n} \frac{n !}{\prod_{t=1}^{p} l_{t} !\left(n-\sum_{t=1}^{p} l_{t}\right) !} f_{l_{1}, \ldots, l_{p}}\left(\bar{x}_{1}, \ldots, \bar{x}_{p}\right) y_{1}^{l_{1}} \ldots y_{p}^{l_{p}} .
\end{aligned}
$$

Подставляя значения $f_{l_{1}, \ldots, l_{p}}\left(\bar{x}_{1}, \ldots, \bar{x}_{p}\right)$ из (3) и (4) и пользуясь полиномиальной теоремой, получаем, что

$$
\begin{aligned}
f\left(\bar{x}_{1}, \ldots, \bar{x}_{p}, y_{1}, \ldots, y_{p}\right)= & \sum_{l_{1}+\ldots+l_{p} \leqslant n} \prod_{k=1}^{d} \sum_{n_{k}^{(1)}, \ldots, n_{k}^{(p)}} \frac{n_{k} !}{\left(n_{k}-\sum_{t=1}^{p} n_{k}^{(t)}\right) ! \prod_{t=1}^{p} n_{k}^{(t)} !} \\
& \quad \times x_{1 k}^{n_{k}^{(1)}} \ldots x_{p k}^{n_{k}^{(p)}} y_{1}^{l_{1}} \ldots y_{p}^{l_{p}} \\
= & \prod_{k=1}^{d}\left(1+x_{1 k} y_{1}+\ldots+x_{p k} y_{p}\right)^{n_{k}} .
\end{aligned}
$$

Для того чтобы из (5) получить производящую функщию $f_{l_{1}, \ldots, l_{p}}\left(\bar{x}_{1}, \ldots, \bar{x}_{p}\right)$ достаточно в функции $f\left(\bar{x}_{1}, \ldots, \bar{x}_{p}, y_{1}, \ldots, y_{p}\right)$ взять коэффициент при

$$
\frac{n !}{\left(n-\sum_{t=1}^{p} l_{t}\right) ! \prod_{t=1}^{p} l_{t} !} y_{1}^{l_{1}} \ldots y_{p}^{l_{p}} .
$$

Полагая в (5) $x_{j k}=x_{j}^{w_{k}}, j=1, \ldots, p$, получим производящую функщию (2) случайных величин $\xi_{1}, \ldots, \xi_{p}$.

Теорема 1 доказана.

Доказательство следствия 1. Производящая функция $f\left(x_{i}, x_{j}, y_{i}, y_{j}\right)$ совместного распределения компонент $\xi_{i}$ и $\xi_{j}$ вектора $\left(\xi_{1}, \ldots, \xi_{p}\right)$ получается из производящей функции $f\left(x_{1}, \ldots, x_{p}, y_{1}, \ldots, y_{p}\right)$, если положить $y_{t}=0$ для $t=1, \ldots, p, i \neq j, t \neq i, j$. Таким образом,

$$
f\left(x_{i}, x_{j}, y_{i}, y_{j}\right)=\prod_{k=1}^{d}\left(1+x_{i}^{w_{k}} y_{i}+x_{j}^{w_{k}} y_{j}\right)^{n_{k}} .
$$

Производящая функция (5) позволяет получить вид матрицы вторых моментов случайного вектора $\left(\xi_{1}, \ldots, \xi_{p}\right)$. Используя при $i \neq j$ равенство

$$
\mathbf{E} \xi_{i} \xi_{j}=\left.\frac{\left(n-l_{i}-l_{j}\right) !}{n !} \frac{\partial^{l_{i}+l_{j}+2} f\left(x_{i}, x_{j}, y_{i}, y_{j}\right)}{\partial x_{i} \partial x_{j} \partial y_{i}^{l_{i}} \partial y_{j}^{l_{j}}}\right|_{x_{i}=x_{j}=1, y_{i}=y_{l}=0}
$$


и теорему 1 , получаем равенство

$$
\mathbf{E} \xi_{i} \xi_{j}=\frac{l_{i} l_{j}}{n(n-1)}\left(\left(\sum_{k=1}^{d} w_{k} n_{k}\right)^{2}-\sum_{k=1}^{d} w_{k}^{2} n_{k}\right)
$$

Полагая в производящей функции (5) $y_{j}=0, j=1, \ldots, p, j \neq t$, и проводя аналогичные вычисления, получим, что

$$
\mathbf{E} \xi_{t}=\frac{l_{t}}{n} \sum_{k=1}^{d} w_{k} n_{k}
$$

Ковариация $i$-й и $j$-й компонент вектора $\left(\xi_{1}, \ldots, \xi_{p}\right)$ равна

$$
\begin{aligned}
\operatorname{cov}\left(\xi_{i}, \xi_{j}\right) & =\mathbf{E} \xi_{i} \xi_{j}-\mathbf{E} \xi_{i} \mathbf{E} \xi_{j} \\
& =\frac{l_{i} l_{j}}{n(n-1)}\left(\left(\sum_{k=1}^{d} w_{k} n_{k}\right)^{2}-\sum_{k=1}^{d} w_{k}^{2} n_{k}\right)-\frac{l_{i} l_{j}}{n^{2}}\left(\sum_{k=1}^{d} w_{k} n_{k}\right)^{2} .
\end{aligned}
$$

Упрощая выражение (8), получим равенство

$$
\operatorname{cov}\left(\xi_{i}, \xi_{j}\right)=-\frac{l_{i} l_{j}}{n-1} B_{n}, \quad i \neq j
$$

где

$$
B_{n}=\frac{1}{n} \sum_{k=1}^{d} w_{k}^{2} n_{k}-\frac{1}{n^{2}}\left(\sum_{k=1}^{d} w_{k} n_{k}\right)^{2}
$$

Заметим, что

$$
\mathbf{D} \xi_{t}=-\operatorname{cov}\left(\xi_{t}, \eta_{t}\right)
$$

где

$$
\eta_{t}=A_{n}-\xi_{t}, \quad A_{n}=\sum_{k=1}^{d} w_{k} n_{k},
$$

то есть случайная величина $\eta_{t}$ равна сумме оставшихся элементов перестановки $s$ на $n-l_{t}$ местах, не использованных при подсчете случайной величины $\xi_{t}$. Действительно,

$$
\operatorname{cov}\left(\xi_{t}, \eta_{t}\right)=\mathbf{E}\left(A_{n}-\xi_{t}\right)-\mathbf{E} \xi_{t} \mathbf{E}\left(A_{n}-\xi_{t}\right)=-\mathbf{D} \xi_{t}
$$

Таким образом,

$$
\mathrm{D} \xi_{t}=\frac{l_{t}\left(n-l_{t}\right)}{n-1} B_{n}, \quad t=1, \ldots, p
$$

Выражения (9) и (10) позволяют получить вид элементов $p \times p$ матрицы $M_{n}=\left(m_{i j}^{(n)}\right)$ вторых моментов вектора $\left(\xi_{1}, \ldots, \xi_{p}\right)$. Из (9) и (10) следует, что

$$
m_{i j}= \begin{cases}l_{i}\left(n-l_{i}\right) B_{n} /(n-1) & \text { при } i=j, \\ -l_{i} l_{j} B_{n} /(n-1) & \text { при } i \neq j .\end{cases}
$$


Отсюда следует, что корреляционная матрица $R_{n}=\left(\rho_{i j}^{(n)}\right)$ имеет вид, приведенный после следствия 1.

Производящую функцию (2) можно использовать для получения остальных характеристик распределения величин $\xi_{t}, t=1, \ldots, p$, а также для проведения неасимптотических расчетов. Однако для больших значений $d$ и $n$ такие расчеты затруднительны.

Доказательство теоремы 2. Для нахождения предельного распределения вектора $\left(\xi_{1}, \ldots, \xi_{p}\right)$ при $n \rightarrow \infty$ и фиксированном $d$ рассмотрим последовательности $\mathscr{A}_{t}^{\prime}$, $t=1, \ldots, p$, и $\mathscr{B}^{\prime}$ длины $n, \mathscr{A}_{t}^{\prime}=\left\{a_{t, j}^{\prime}\right\}$, где $j=1, \ldots, n$,

$$
a_{t, j}^{\prime}= \begin{cases}\left(1-l_{t} / n\right) / n, & \text { если } j=i_{1}^{(t)}, \ldots, i_{l_{t}}^{(t)}, \\ -l_{t} / n^{2} & \text { для остальных } j,\end{cases}
$$

и $\mathscr{B}^{\prime}=\left\{b_{j}^{\prime}\right\}, j=1, \ldots, n$, где

$$
b_{j}^{\prime}=\frac{1}{n}\left(w_{m}-\frac{1}{n} \sum_{k=1}^{d} w_{k} n_{k}\right), \quad j=\sum_{k=1}^{m-1} n_{k}+1, \ldots, \sum_{k=1}^{m} n_{k}, \quad m=1, \ldots, d,
$$

причем предполагается, что $\sum_{k=1}^{0} n_{k}=0$.

Определим случайные величины $\xi_{1}^{\prime}, \ldots, \xi_{p}^{\prime}$, полагая

$$
\xi_{t}^{\prime}=\sum_{i=1}^{n} a_{t, i}^{\prime} b_{x(i)}^{\prime}, \quad t=1, \ldots, p
$$

где $x(i)$ - образ элемента $i$ при случайном отображении $x$ из симметрической группы $S_{n}$, при этом предполагается, что на группе $S_{n}$ задано равновероятное распределение.

Введенные выше случайные величины $\xi_{t}$ линейно выражаются через случайные величины $\xi_{t}^{\prime}$ следующим образом:

$$
\xi_{t}=n^{2} \xi_{t}^{\prime}+\frac{l_{t}}{n} \sum_{k=1}^{d} w_{k} n_{k}, \quad t=1, \ldots, p
$$

Соотношение (11) легко получить, учитывая, что случайные величины $\xi_{t}$ можно представить в виде

$$
\xi_{t}=\sum_{i=1}^{n} a_{i}^{(t)} b_{x(i)}
$$

где последовательности $\mathscr{A}_{t}=\left\{a_{j}^{(t)}\right\}, t=1, \ldots, p$, и $\mathscr{B}=\left\{b_{j}\right\}, j=1, \ldots, n$, имеют вид

$$
\begin{aligned}
a_{j}^{(t)} & = \begin{cases}1 & \text { при } j=i_{1}^{(t)}, \ldots, i_{l}^{(t)}, \\
0 & \text { для остальных } j,\end{cases} \\
b_{j} & =w_{m}, \quad j=\sum_{k=1}^{m-1} n_{k}+1, \ldots, \sum_{k=1}^{m} n_{k}, \quad m=1, \ldots, d .
\end{aligned}
$$


Сначала найдем предельное распределение вектора $\left(\xi_{1}^{\prime}, \ldots, \xi_{p}^{\prime}\right)$. Для этого воспользуемся теоремой 2 из [2]. Поскольку

$$
\begin{aligned}
n \sum_{j}\left(a_{t, j}^{\prime}\right)^{2} & =\frac{l_{t}}{n}\left(1-\frac{l_{t}}{n}\right), \\
n \sum_{j}\left(b_{j}^{\prime}\right)^{2} & =\frac{1}{n} \sum_{k=1}^{d} w_{k}^{2} n_{k}-\frac{1}{n^{2}}\left(\sum_{k=1}^{d} w_{k} n_{k}\right)^{2},
\end{aligned}
$$

при $n \rightarrow \infty, l_{t} / n \rightarrow \alpha_{t}$, где $\alpha_{t}$ фиксировано, $t=1, \ldots, p$, и при существовании пределов

$$
\lim _{n \rightarrow \infty} \frac{1}{n} \sum_{k=1}^{d} w_{k} n_{k}=A, \quad \lim _{n \rightarrow \infty}\left(\frac{1}{n} \sum_{k=1}^{d} w_{k}^{2} n_{k}-\frac{1}{n^{2}}\left(\sum_{k=1}^{d} w_{k} n_{k}\right)^{2}\right)=B, \quad B>0,
$$

условия теоремы 2 из [2] выполняются и, следовательно, вектор $\left(\xi_{1}^{\prime}, \ldots, \xi_{p}^{\prime}\right)$ имеет асимптотически нормальное распределение, что доказывает теорему 2.

В заключение отметим, что результаты работы [2] позволяют также найти параметры предельного распределения случайного вектора $\left(\xi, \ldots, \xi_{p}\right)$ в случае, когда условие (1) не выполняется.

\section{Список литературы}

1. Сачков В. Н., Комбинаторные методы дискретной математики. Наука, Москва, 1977.

2. Колчин В. Ф., Чистяков В. П., О предельных распределениях одной статистики. Теория вероятностей и ее применения (1974) 19, №2, 367-373.

3. Лапшин А. В., Распределение сумм элементов случайной $n$-перестановки одного класса. Обозрение прикладной и промышленной математики (1997) 4, №3, 370-372.

Статья поступила 10.02.2005. 